\title{
Proposta de Planejamento de Medições em Projetos de Software Utilizando uma Ferramenta de Modelagem
}

\author{
Fabrício M. da Silva ${ }^{1}$, Odisnei Galarraga ${ }^{1,2}$ \\ ${ }^{1}$ Pós Graduação em Qualidade de Software - Universidade do Vale do Rio dos Sinos \\ (UNISINOS) \\ Av. Unisinos, 950 - Bairro Cristo Rei - CEP:93022-000 - São Leopoldo - RS - Brazil \\ ${ }^{2}$ Software Process Consultoria \\ machadodasilva@gmail.com, Odisneidswprocess.com.br
}

\begin{abstract}
This paper presents a proposal of documentation of all components involved in the planning of measurements in a software company, by means of graphical representations that cover the various levels of abstraction of these components, using a modeling tool. The generated documentation also comprises a measurement plan, and was made available in a browsable format on the company intranet, serving as an important form of planning measurements communication to all interested parties.
\end{abstract}

Resumo. Este artigo apresenta uma proposta de documentação de todos os componentes envolvidos no planejamento de medições em uma empresa de software, por meio de representações gráficas que cobrem os vários níveis de abstração desses componentes, utilizando uma ferramenta de modelagem. A documentação gerada engloba ainda um Plano de Medições, e foi disponibilizada em um formato navegável na intranet da empresa, servindo como importante forma de comunicação do planejamento das medições a todos os interessados.

\section{Introdução}

Uma das maiores dificuldades de uma organização de software, ao definir um processo de medição, é fazer com que todos os envolvidos nessa definição entendam como o processo de medição foi estruturado até chegar à sua parte mais visível, que são justamente as medidas e os indicadores identificados.

Os modelos de referência, como o MR-MPS Modelo de Referência - Melhoria de Processos do Software Brasileiro (Rocha, A.R.C.; Souza, G.S..2 2011) e o CMMI Capability Maturity Model Integration (Chrissis, M.B. et al., 2011), orientam o processo de medições no sentido de um planejamento com base em uma abordagem topdown. Essa abordagem propõe a identificação de necessidades de informações a partir dos níveis gerenciais da organização, de forma alinhada com os objetivos estratégicos e de negócio. A partir dessas necessidades de informações gerenciais, são estabelecidos objetivos de medição, os quais levam ao desdobramento de medidas e indicadores necessários para atender esses objetivos. 
Para facilitar o entendimento de todos os envolvidos no planejamento da medição a respeito desses vários níveis de abstração presentes no processo, surgiu a ideia do apoio de uma ferramenta de modelagem já utilizada pela organização na engenharia de software dos seus produtos. A ferramenta foi escolhida porque os recursos humanos da organização já possuíam o conhecimento técnico necessário e porque ela permite a criação de diagramas do tipo mind maps (mapas mentais), considerados apropriados para a representação dos níveis de abstração do processo de medição.

A proposta da utilização da ferramenta de modelagem ganhou o apoio de todos e assim o plano de medição foi totalmente estruturado dentro da ferramenta, em substituição aos tradicionais documentos textuais que são comuns nesse tipo de atividade.

Na seção 2 deste artigo é apresentado o embasamento teórico, descrevendo melhor o conceito de medidas, indicadores, técnica GQM e a ferramenta de modelagem utilizada neste caso. A seguir, na seção 3, é apresentada a proposta de utilização da ferramenta de modelagem aplicada a um caso real. A seção 4 apresenta as conclusões do trabalho. A seção 5 finaliza este artigo com as referências bibliográficas utilizadas.

\section{Conceitos e Embasamento Teórico}

A cada dia que passa os softwares tornam-se mais complexos, sendo utilizados nos mais diversos ambientes. Os processos de desenvolvimento do software devem acompanhar esse crescimento, e o gerenciamento desses processos tornam-se, assim como o seu produto final, cada vez mais complexos.

Diante desse cenário, conhecer e identificar os pontos falhos em processos de software torna-se um desafio cada vez maior para os gestores. Nesse contexto, definir e institucionalizar um processo de Medição é fundamental para auxiliar a identificação desses pontos de melhorias.

O PSM - Practical Software and Systems Measurement (Jones, C. et al, 2003), define que medição em projetos de software deve servir como uma poderosa ferramenta aos gestores, e quando integrada a todos os processos que fazem parte do desenvolvimento do software ajuda os gerentes a identificarem riscos, problemas específicos e o impacto desses problemas.

Os indicadores disponibilizados a partir de um processo de medição, quando alinhados aos objetivos estratégicos e de negócio, ajudam os gestores de uma organização a saber se esta segue os rumos definidos no planejamento estratégico. Os benefícios de um processo de medição bem planejado e alinhado com as necessidades de informação daqueles que fazem parte do processo de desenvolvimento estão principalmente relacionados a conhecimento e respostas a perguntas. Parte fundamental de qualquer processo de medição, as medidas e indicadores são peças fundamentais para a obtenção dos efetivos resultados a que este processo se destina. Por isso, é imprescindível definir um conjunto de medidas que atendam a todos os objetivos de medição e estas devem servir de base para os indicadores.

O PSM define que os 9 princípios básicos da medição são:

- Use questões e objetivos orientados aos requisitos de medição;

- Defina e colete medidas baseadas em técnica e processos de gerenciamento; 
- Colete e analise dados ao nível de detalhamento capaz de identificar e isolar problemas;

- Implemente uma capacidade de análise independente;

- Use um processo de análise sistemático para rastrear a medida com a decisão;

- Interprete os resultados da medição no contexto de outros projetos de informação;

- Integre a medição ao processo de gerenciamento de projetos através do ciclo de vida;

- Use o processo de medição como uma base para os objetivos de comunicação;

- Foque inicialmente na análise ao nível de projeto.

As medidas coletadas são a base para os indicadores. Um indicador é uma medida ou uma combinação de medidas que provê uma compreensão acerca de algo que desejamos conhecer. Sua finalidade é quantificar e ajudar a identificar o status de projetos de software com relação aos seus objetivos.

Um indicador pode permitir identificar desvios no comportamento de um processo a partir de limites estabelecidos de acordo com o que está sendo analisado. Além disso, deve descrever como deve ser analisado para que seja possível realizar uma correta interpretação, além de identificar fatores que podem justificar determinados resultados inesperados.

Algumas técnicas são utilizadas para auxiliar a identificação de medidas e indicadores, dentre as quais destacamos a SMART Specific. Measurable. Attainable. Result oriented. Time-bound GQM The Goal Question Metric Approach (Basili, V.R.; Caldiera, G; Rombach, H.D., 2004). A técnica S.M.A.R.T define que:

- Um objetivo específico é mais fácil de ser atingido e objetivos genéricos podem não estar claros para o time;

- Deve ser estabelecido um critério concreto para medir o progresso do objetivo definido;

- À medida que o time define os objetivos inicia também a análise do que é necessário para atingi-los;

- Para ser orientado a resultado, o objetivo deve estar conectado à visão estratégica da organização. Se este estiver desconectado, há grande chance de perder prioridade;

- Objetivos devem ser delimitados por tempo. Sem limite de tempo definido não existe senso de urgência ou foco.

A técnica GQM, - pode ser usada para definir o conjunto de medidas a serem coletadas. Essa técnica baseia-se no fato de que deve existir uma necessidade clara associada a cada medida.

A GQM inicia-se com a identificação dos interessados na medição. Com base nos interessados, estabelecem-se os principais objetivos de medição para a organização, o projeto ou uma tarefa específica. A partir dos objetivos, devem ser geradas perguntas cujas respostas dirão se os objetivos foram ou não alcançados. 
A partir das perguntas, definem-se medidas, que dados serão necessários, quais os formatos, como coletar e onde armazenar. A Figura 1 ilustra como está estruturada a técnica GQM.

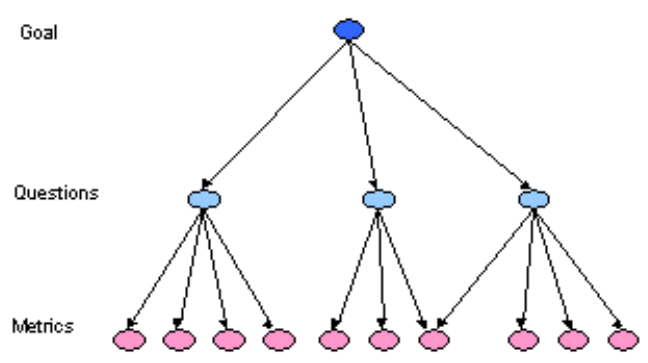

Figura 1. Estrutura da técnica GQM

O Planejamento da Medição utilizando uma técnica como GQM tradicionalmente é implementado através do uso de documentação textual, mas a utilização de uma ferramenta de modelagem, além de possível, também traz os benefícios da representação visual. Uma ferramenta de modelagem que está sendo muito utilizada nas organizações de desenvolvimento de software, por ser considerado um excelente custo benefício, é o Enterprise Architect popularmente chamado de EA.

O EA pode ser utilizado como ferramenta de modelagem em todo o ciclo de vida do desenvolvimento de software. Além disso, a ferramenta também disponibiliza alguns plug-ins onde é possível utilizá-la para outros fins como, por exemplo, modelagem de processos de negócio com BPM (Business Process Modeling) e a utilização para a representação de Mind Maps.

\section{Proposta de Planejamento de Medições em Projetos de Software}

O Planejamento de um processo de medição bem alinhado com os objetivos estratégicos foi a ideia que norteou a definição do processo de medição para uma empresa de software localizada no Rio Grande do Sul. A empresa é uma fábrica de software que atua como fornecedora de soluções de software principalmente para a área calçadista. As suas atividades estão ligadas a suporte e evolução dos seus produtos, que são customizados de acordo com a necessidade de negócio de seus clientes. A empresa trabalha com produtos desenvolvidos em tecnologia Java, Power Builder e Cold Fusion. A preocupação, desde o início da definição de seu processo de medição, e também de acordo com o modelo de referência seguido (MR-MPS), era de um processo extremamente alinhado com os objetivos estratégicos da organização. Pensando nisso, algumas ações foram adotadas. Dentre elas, podemos citar:

- Contratação de apoio de consultoria especializada;

- Dedicação integral de um time de melhoria de processos;

- Envolvimento direto do time de melhoria de processos com os gestores.

O primeiro grande desafio surgiu logo no início, e foi justamente na definição de quais seriam os objetivos de medição, uma vez que cada gestor defendia uma necessidade 
específica de informação que seria interessante no seu contexto, mas que muitas vezes não era interessante para a organização como um todo.

Foi nesse momento que se optou por adotar a técnica de GQM para auxiliar o time no estabelecimento de objetivos de medição, medidas e indicadores alinhados aos objetivos de negócio e estratégicos da organização.

O uso da técnica de GQM tornou-se tão empolgante para os envolvidos, que nesse momento surgiu outra ideia: usar o apoio de uma ferramenta de modelagem para deixar clara a representação de como se chegou até os objetivos de medição. A organização já tinha experiência em trabalhar com a ferramenta de modelagem EA para processos de engenharia de software e análise de negócio. Logo, a ferramenta foi a escolhida.

Como se tratava de uma representação de como ideias estavam interligadas em um desdobramento de objetivos de medição, foi adotado o plugin de mind map da ferramenta EA para a realização desse trabalho. Usando mind map foi possível criar a primeira visão dos objetivos de medição e seus relacionamentos com objetivos estratégicos e as necessidades de informação, conforme Figura 2.

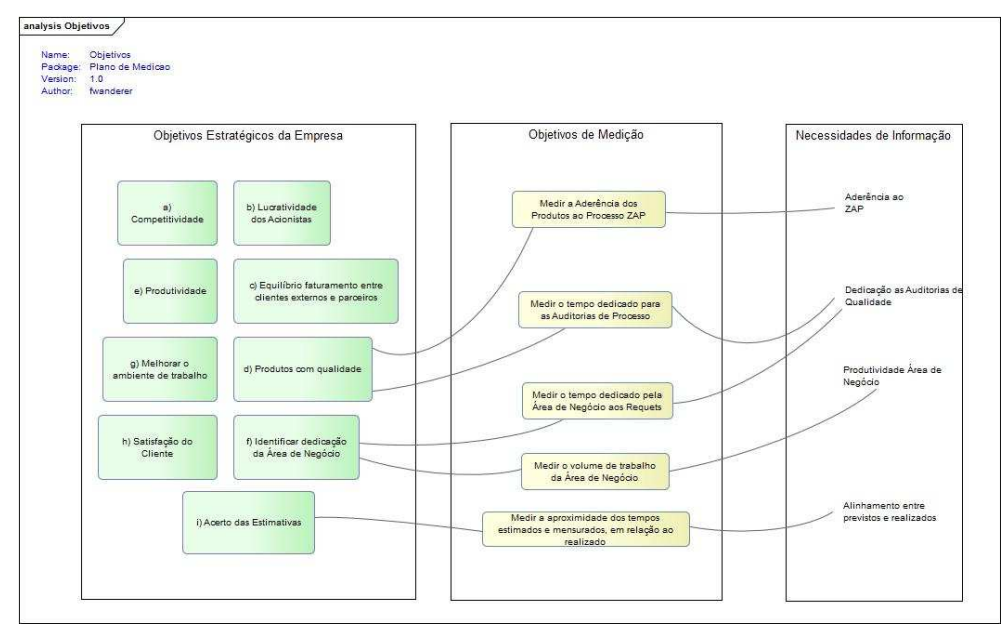

Figura 2. Relacionamento dos objetivos de medição

O uso desse tipo de representação através de uma ferramenta de modelagem possibilitou um maior entendimento entre os envolvidos na definição do processo de medição, pois a forma de representação visual permitiu a todos enxergar claramente a relação dos objetivos de medição com os demais níveis de abstração envolvidos.

A ideia de representação através da ferramenta de modelagem foi progressivamente ganhando adesão, e a adoção dessa técnica para todo o planejamento de medição acabou por tornar-se consenso na organização. $O$ passo seguinte foi usar uma segunda visão dentro do modelo, onde seriam representados os relacionamentos dos objetivos de medição com as medidas e indicadores do Plano de Medição.

Para a identificação das medidas e indicadores foi utilizada a técnica de GQM, conforme citado anteriormente. Porém, a tarefa de alinhar o conhecimento de todos os envolvidos com a técnica de GQM não foi muito fácil, assim como mostrar claramente o relacionamento de medidas e indicadores utilizando-se documentação textual. A 
Figura 3 mostra a visão criada para representar a utilização da técnica de GQM derivando medidas e indicadores a partir de objetivos de medição identificados anteriormente.

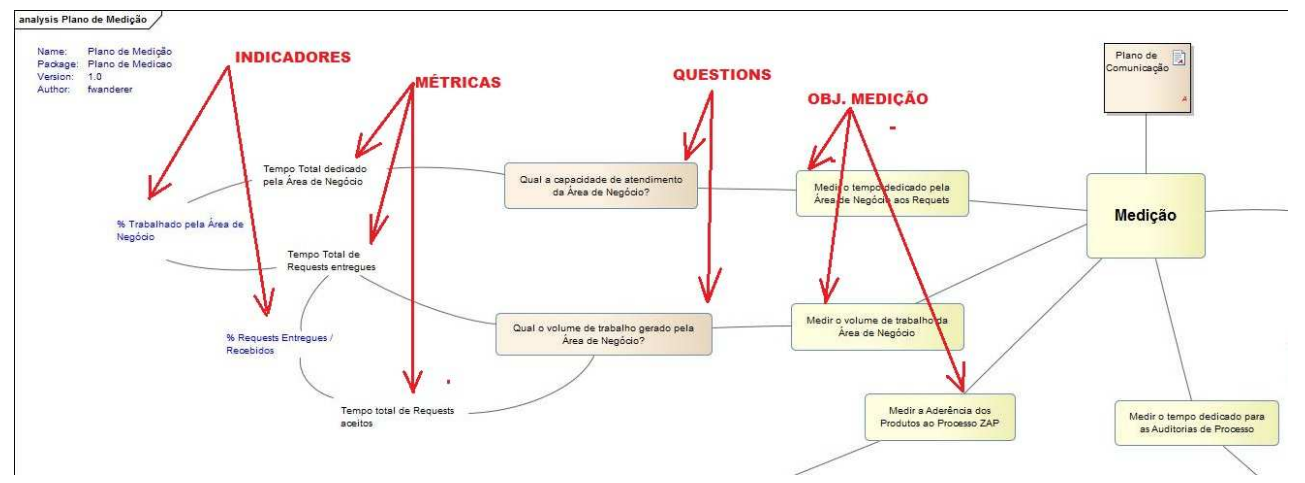

Figura 3. Plano de medição

A utilização dessa visão facilitou e alinhou o entendimento entre todos os envolvidos com relação à identificação das medidas e indicadores que estariam no Plano de Medição organizacional, e também serviu como importante ferramenta de aprendizado da técnica de GQM. Outra visão criada nesse modelo foi uma representação das responsabilidades pela coleta das medidas. Para isso, a ideia foi a utilização de elementos visuais do modelo de Casos de Uso (atores) representando os papéis responsáveis pelas coletas. Para isto, foi criada outra visão conforme mostra a Figura 4.

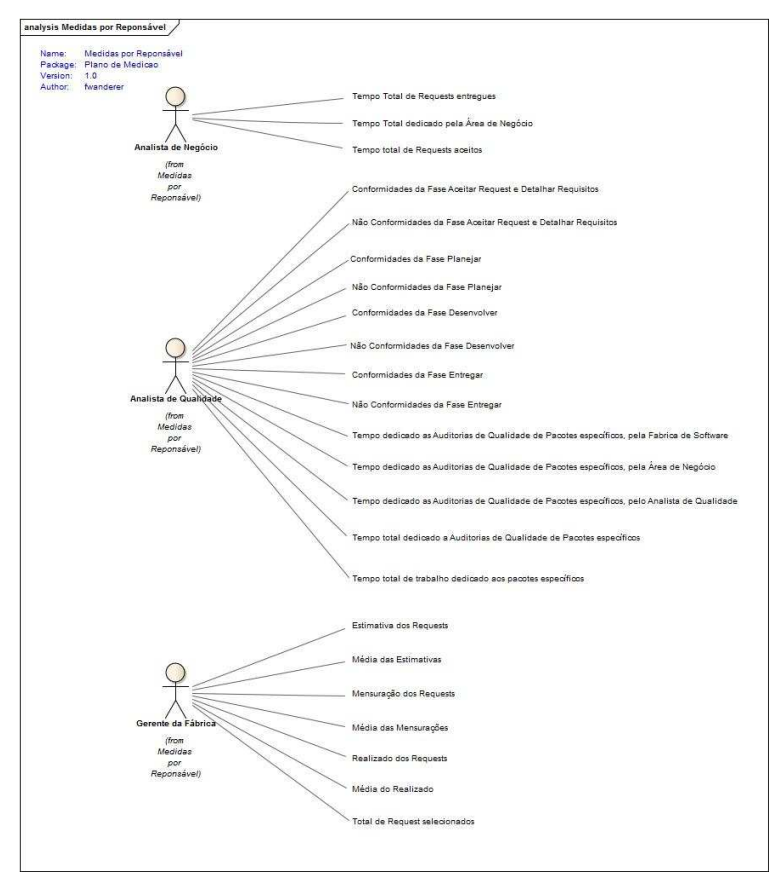

Figura 4. Responsável por medida. 
Através da ferramenta, outros recursos, como a geração de HTML de modelos, possibilitaram a publicação do Plano de Medição e sua navegação por meio da intranet organizacional. Desta forma, todo o planejamento de medição da organização foi migrado para a ferramenta de modelagem, eliminando os tradicionais documentos textuais que normalmente são adotados nesses casos. Com isto tirou-se proveito da facilidade da utilização de uma representação visual para apresentação dos diversos níveis de abstração envolvidos na elaboração de um Plano de Medição. A Figura 5 mostra a publicação do Plano de medição na intranet organizacional e a possibilidade do usuário navegar pelas medidas e indicadores para obter mais detalhes sobre eles. $\mathrm{Na}$ imagem são apresentados maiores detalhes do indicador "\% trabalhado pela área de negócio".

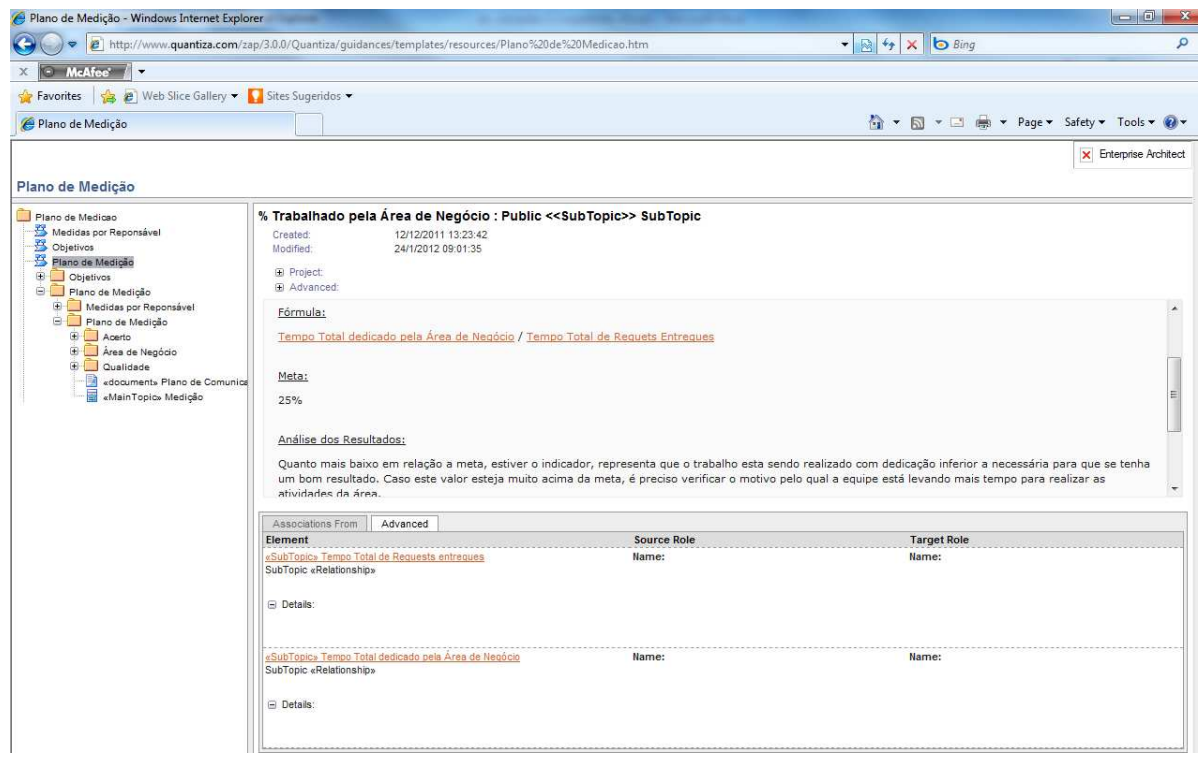

Figura 5. Publicação do Plano de Medição

Outros ganhos foram percebidos, como a possibilidade de geração de uma publicação textual do Plano de Medição, caso fosse necessário, através do recurso de geração de documentação da própria ferramenta, além da utilização de cores para diferenciar, por exemplo, medidas propostas e medidas já aprovadas para o Plano de Medição. Isto tudo somado à facilidade de manutenção do Plano.

\section{Conclusões}

Tradicionalmente, todo o planejamento de um processo de medição é feito utilizando-se documentação textual. Ter o apoio de uma ferramenta de modelagem possibilitou ganho no entendimento de como o plano de medição está estruturado, facilitando a manutenção desse documento e ainda usufruindo de benefícios que a ferramenta de modelagem possui, como por exemplo, geração de HTML e documentação textual, se esta for necessária. $\mathrm{O}$ uso de uma ferramenta de modelagem para apoiar o planejamento da medição também possibilitou deixar mais clara a rastreabilidade entre todos os níveis de abstração de um plano de medição, o que permitiu a todos enxergar por que uma medida ou indicador está sendo utilizado. 


\section{Referências Bibliográficas}

Jones, C. et al. Practical Software and Systems Measurement: A Foundation for Objective Project Management. Department of Defense and US Army, 2003.

Basili, V.R.; Caldiera, G; Rombach, H.D. The Goal Question Metric Approach

Goethert, W.; Siviy, J. Applications of the Indicator Template for Measurement and Analysis. Pittsburgh: Carnegie Mellon University, 2004.

Rocha, A.R.C.; Souza, G.S. Guia de Implementação - Parte 2: Fundamentação para Implementação do nível F do MR-MPS. SOFTEX, 2011.

Chrissis, M.B. et al. CMMI for Development - Guideline for Process Integration and Product Improvement. 2011.

Berry, S.; Thomas R. - Use SMART Objectives To Focus Goals, Plans and Performance. 2008. 